\title{
ALD TITANIUM NITRIDE ON VERTICALLY ALIGNED CARBON NANOTUBE FORESTS FOR ELECTROCHEMICAL SUPERCAPACITORS
}

\author{
Emmeline Kao, Chen Yang, Roseanne Warren, Alina Kozinda, Liwei Lin \\ Department of Mechanical Engineering, \\ Berkeley Sensor and Actuator Center \\ University of California, Berkeley, CA 94720 \\ Corresponding author \\ Emmeline Kao \\ kao@berkeley.edu
}

(631) 2756976

\begin{abstract}
Titanium nitride (TiN) coating on vertically aligned carbon nanotube (VACNT) forest electrodes by means of atomic layer deposition (ALD) to store charge in electrochemical supercapacitors has been demonstrated. Experimental results show that after 400 cycles of ALD process, a roughly $20 \mathrm{~nm}$-thick TiN layer is coated uniformly on individual CNTs in the forest electrodes. The electrochemical characterizations show 2000 times higher capacitance of the ALD TiN-CNT device as compared to that of a flat-shape TiN electrode. Furthermore, over 500\% enhancement of electrochemical capacitance of the ALD TiN-CNT device ( $\left.81 \mathrm{mF} / \mathrm{cm}^{2}\right)$ as compared to bare CNT forest electrodes $\left(14 \mathrm{mF} / \mathrm{cm}^{2}\right)$ has been accomplished due to the increased oxygen vacancies on the TiN surfaces. As such, this work presents a new path to increase energy density of supercapacitors using TiN-based porous electrode materials.
\end{abstract}

Keywords: Titanium nitride; supercapacitor; atomic layer deposition; electrochemistry; CNT; VACNT 


\section{INTRODUCTION}

Electrochemical supercapacitors hold a unique position in energy storage applications because of their high power density, which translates to quick charge/discharge cycles and potential for high stability. However, the key-limiting factor in supercapacitors is their lower storage capacity relative to that of batteries or other high energy density storage devices, which arises from the charge storage mechanisms used in supercapacitor devices. That is, since supercapacitors store charge through the electric double-layer, they have the ability to charge and discharge quickly, as ions from the electrolyte adsorb on and desorb from the surface of the electrode. Because the number of ions that can gather at the surface is directly proportional to the electrode surface area, the energy storage capabilities of electrodes can be increased through the texturization of electrode surfaces. Improvements in capacitance due to the electric double-layer are often achieved by using high surface area materials with high intrinsic capacitances, such as porous carbon, activated carbon, or vertically-aligned carbon nanotubes [1,2]. These supercapacitors charge and discharge quickly, as the charge is highly mobile and the charge layer forms only at the surface of the material. In contrast, lithium-ion batteries have relatively slow charge/discharge cycles, as ions must intercalate themselves into the material. Moreover, during the process of intercalation, electrodes may become susceptible to damage. For example, silicon, known as one of the highest energy density lithium-ion battery materials, can expand up to $400 \%$ upon intercalation [3]. In contrast, damage in double-layer supercapacitors is minimal, since charge simply gathers on the surface and does not insert itself into the electrode area.

The second mechanism of charge storage in an electrochemical supercapacitor is achieved by a Faradaic redox reaction occurring at the surface of the electrode, referred to as pseudocapacitance. In contrast to intercalation processes, these processes are much faster since they occur only on the surface layers of the electrode. However, many pseudocapacitors suffer from poor stability, since electrodes may experience damage from ion insertion. These two mechanisms of supercapacitor charge storage, when employed in tandem, can dramatically increase charge storage capacity, depending on the device architecture and mode of pseudocapacitance. For example, prior works using conductive polymers [4], nickel nanoparticles [5], or $\mathrm{ALD} \mathrm{RuO}_{2}$ loading [6] onto VACNT forest electrodes have shown up to 170 times higher capacitances by employing pseudocapacitance. However, the resulting polymer and nickel depositions are not IC compatible and have limited enhancements in capacitance, while the $\mathrm{RuO}_{2}$ is expensive and not suitable for large-scale production. Here, we examine the possibility of using titanium nitride as an alternative material for MEMS supercapacitor applications, since TiN is a standard material in IC processes and processing techniques and applications in ICs for TiN have been well explored.

At the macro scale, titanium nitride films have been known to be physically stable, with exceptional hardness, and are thus widely used as protection coatings in tool 
manufacturing. Although chemically classified as a ceramic, titanium nitride has good electrical conductivity and serves well as diffusion barriers for electronic circuitry. Recently, TiN has been studied for energy storage devices, such as capacitors [7] and battery electrodes [8,9], because of its good electrical conductivity and electrochemical performance.

For applications in electrochemical supercapacitors, control over deposition upon irregular surfaces is central to optimizing performance. However, previous titanium nitride supercapacitors have been limited in geometry or surface functionalization due to the difficulty of precise control in the fabrication process. These electrodes have previously been fabricated through either a two-step, high temperature process, which limits selection of high surface area electrode materials $[10,11]$, or by sputtering with poor conformality and coverage [12]. Here we present results from depositions of angstrom-level precision ALD TiN onto porous CNT forest electrodes, which demonstrate outstanding performance in the supercapacitor electrode applications.

\section{Design, FABRication, AND MEASUREMENT}

In our supercapacitor device, we increase double-layer capacitance by using a vertically aligned carbon nanotube (VACNT) forest, a well-known, porous, and high surface area device structure. We then further increase charge capacitance by conformally coating the CNT forest with titanium nitride (TiN), which has previously been shown to facilitate Faradaic redox surface reactions due to increased oxygen vacancies at the native oxide surface (see Figure 1) [13]. This increased capacitance is achieved by: (1) increasing the surface area using the porous CNT forest architecture and (2) allowing for redox reactions to occur in the oxygen vacancy-rich TiN surface layer. The large surface area increases the electric double-layer capacitance as storage/release of electrical charges occurs by the adsorption/desorption of charges on the surface of TiN. The surface layer oxidizes, forming a native oxide layer of $\mathrm{TiO}_{2-\mathrm{x}} \mathrm{N}_{\mathrm{x}}$ with high nitrogen concentration and a large number of oxygen vacancies for Faradaic electrochemical storage, enhancing energy storage capabilities through pseudocapacitance.

\section{Electrode Preparation}

The electrode is prepared as shown in Figure 2. First, a silicon substrate is prepared with $100 \mathrm{~nm}$ of thermally grown oxide. Next, a thin layer of molybdenum is deposited by electron-beam evaporation to form a conductive layer. The catalyst is a thermally evaporated mixture of $5 \mathrm{~nm}$ iron and $5 \mathrm{~nm}$ aluminum; the aluminum prevents molybdenum and iron from forming an alloy. The substrate is transferred to a vacuum quartz tube furnace to grow the CNT forest by chemical vapor deposition (CVD) [14]. Once the temperature of the furnace stabilizes at $720^{\circ} \mathrm{C}$, the process gases (hydrogen and ethylene) are introduced in a 7:1 ratio with respect to volumetric flow rate. When heated to 
high temperatures, the catalysts nucleate and form droplets on the conductive surface, forming the basis for CNT forest growth. At this high temperature, when placed in an ethylene-rich environment, the CNTs are then grown by CVD. The carbon nanotubes as grown by CVD are multi-walled nanotubes and therefore metallic in nature.

ALD is a CVD process by which self limiting reactions occur between sequentiallypulsed, gas phase precursors. TiN is deposited by ALD using two precursors: Tetrakis (dimethylamido) titanium (TDMAT) and nitrogen plasma. The process is highly dependent on ALD configuration, temperature, precursors, and the presence of atmospheric contaminants. ALD deposition was conducted using an ALD specific tool as sold by Cambridge Nanotech (model Fiji). ALD deposition was tested for $200{ }^{\circ} \mathrm{C}, 250{ }^{\circ} \mathrm{C}$, and 300

${ }^{\circ} \mathrm{C}$, as shown in Figure 3a. Ultimately, TiN films deposited at $250{ }^{\circ} \mathrm{C}$ were used, as in Cambridge Nanotech's standard TiN recipe. Additionally, films deposited at $250{ }^{\circ} \mathrm{C}$ show the lowest oxygen-to-titanium ratio, indicating a higher amount of TiN in the sample. TDMAT heated to $75^{\circ} \mathrm{C}$ was pulsed for $0.25 \mathrm{~s}$, and $\mathrm{N}_{2}$ plasma pulsed for $20 \mathrm{~s}$ at an overall chamber temperature of $250{ }^{\circ} \mathrm{C}$, with purge steps of $6 \mathrm{~s}$ and $5 \mathrm{~s}$, respectively, between the precursor steps. The pressure was on the order of millitorrs. The growth rate is roughly 6 $\AA$ Acycle. The as-deposited film forms a native oxide in the presence of atmosphere, which is exacerbated in high-temperature environments. Therefore, the deposited film is left to cool for 30 minutes - 1 hour under vacuum environment.

\section{RESULTS AND DiSCUSSION}

Figure $3 \mathrm{~b}$ shows SEM images of a vertically aligned CNT forest coated with ALD TiN. As the images show, a CNT forest of roughly $30 \mu \mathrm{m}$ in height was successfully grown, corresponding to 20 minutes of growth time in an ethylene environment. As shown in the close-up SEM image (Figure 3c), the TiN coverage is conformal in the CNT forest. Figures $3 \mathrm{~d}$, 3e show TEM images of bare carbon nanotubes and TiN-coated carbon nanotubes. The bare carbon nanotube shows a hollow center and multiple walls, corresponding with the multiwalled nature of the CVD grown CNTs. Figure 3e shows conformal coating and uniform thickness of the ALD TiN on CNTs, corresponding to 400 cycles of ALD. The CNT has a diameter of roughly $20 \mathrm{~nm}$ and the TiN is roughly $20 \mathrm{~nm}$ in thickness, consistent with the growth rate for this TiN recipe.

\section{Electrode Testing}

The electrode was tested using a three-electrode setup in aqueous and organic electrolyte. Electrodes were used as prepared and tested with Pt wire as the counter electrode. In aqueous $0.5 \mathrm{M} \mathrm{H}_{2} \mathrm{SO}_{4}$ solution, measurements were taken vs. an $\mathrm{Ag} / \mathrm{AgCl}$ reference electrode (see Figure 4a). In non-aqueous 0.1 M Tetrabutylammonium hexafluorophosphate solution (dissolved in acetonitrile), measurements were taken vs. an Ag+ reference electrode. 
TiN-CNT, CNT, and planar TiN electrodes were tested using a three-electrode setup with $0.5 \mathrm{M} \mathrm{H}_{2} \mathrm{SO}_{4}$ as electrolyte, $\mathrm{Pt}$ wire as counter electrode, vs. $\mathrm{Ag} / \mathrm{AgCl}$ as shown in Figure $4 \mathrm{a}$. The cyclic voltammetry curves show an improvement of $>500 \%$ in capacitance of the TiN-CNT device relative to that of bare CNTs $\left(81 \mathrm{mF} / \mathrm{cm}^{2}\right.$ for TiN-CNT and 14 $\mathrm{mF} / \mathrm{cm}^{2}$ for CNT electrodes), and the bare CNTs show an almost 400x improvement in capacitance relative to planar TiN electrodes $\left(0.2 \mathrm{mF} / \mathrm{cm}^{2}\right)$.

The significance of surface area on supercapacitor charge storage is shown in Figure $4 \mathrm{~b}$, wherein using a porous CNT framework increases capacitance almost 400x over that of a planar TiN electrode. Furthermore, the improvement of $>500 \%$ in capacitance by conformal coating of TiN indicates that charge storage in the TiN-CNT device does not only take place via ion adsorption/desorption but also through Faradaic redox reactions, or pseudocapacitance. By increasing surface area, one can drastically improve supercapacitor performance. In contrast to current electrochemical supercapacitor work using TiN, ALD allows for robust coating, conformal coating, and angstrom level precision, providing both great flexibility in choice and architecture of the device and potential for increasing surface area.

Figure 5a shows electrochemical impedance spectroscopy of the TiN-CNT electrode, with two clear regimes of double-layer capacitance at high frequencies and diffusionlimited capacitance at lower frequencies, corresponding to the electric double-layer storage mechanism and pseudocapacitive storage mechanism, respectively. The Randle's cell (Figure 5a inset) represents a first-order equivalent circuit approximation of an adsorption/desorption and diffusion model that has been fitted to experimental data, showing the trends of storage mechanisms in the device. In the model, adsorption is represented by a constant phase element, corresponding to double-layer capacitance, and pseudocapacitance is represented by the Warburg impedance, a diffusion-limited impedance. The Randle's cell fit for the electrode in aqueous solution is shown in Figure $5 b$ $5 \mathrm{c}$ and show poor agreement in the low-frequency range because, in practice, Warburg impedance is not ideal. In the low-frequency (diffusion-dominated) regime, behavior in the model is determined largely by the Warburg impedance, which is modeled as ideal. In practice, while diffusion behavior can be approximated by ideal Warburg impedance, behavior in porous pseudocapacitive electrodes have shown to have dependencies on conductance of the solution and properties of the porous electrode structure [15].

Pseudocapacitive effects most likely arise due to increased oxygen vacancies within the native $\mathrm{TiO}_{2-\mathrm{x}} \mathrm{N}_{\mathbf{x}}$ layer that forms upon exposure to the atmosphere. As seen in Figure 3a, the oxygen content in the surface of the electrode, as measured using XPS, is relatively high, implying a high-oxygen concentration in the native oxide layer. Furthermore, nitrogen doping of titanium dioxide materials has been shown to increase oxygen vacancies, thus increasing the potential for charge insertion and storage. The ability to store more charge results from the reorganization of electrons associated with the absent oxygen. The defect state has been shown to be introduced at approximately $0.8 \mathrm{eV}$ below the valence band 
[16], implying that the electrons are fixed, not mobile. These electrons are transferred into empty $3 \mathrm{~d}$ orbitals belonging to adjacent titanium atoms, resulting in a change of formal oxidation state from $\mathrm{Ti}^{+4}$ to $\mathrm{Ti}^{+3}[17]$.

In testing pseudocapacitive devices, stability must be considered, since damage to the electrode can occur from the insertion of ions, or damage to the electrode in the case of non-reversible Faradaic reactions. Over continuous charge/discharge cycles, the device is relatively stable. However, as can be seen in Figure $4 \mathrm{~b}$, the reaction is not completely reversible. We hypothesize that this is due to irreversible oxidation at the surface of the TiN electrode. Existing work on TiN supercapacitor devices has shown relatively stable oxygen vacancies in the presence of non-reversible oxidation due to simultaneous diffusion of nitrogen from the TiN bulk into the native oxide layer [12]. In the proposed system, the native oxide layer maintains the same number of oxygen vacancies, as the oxidation occurs through diffusion of oxygen into the bulk while nitrogen simultaneously diffuses from the bulk to the surface layer.

To increase stability, the electrode was tested in an oxygen-free electrolyte of $0.1 \mathrm{M}$ Tetrabutylammonia hexafluorophosphate dissolved in acetonitrile. As shown in Figure 6a, performance decreases relative to the electrode tested in sulfuric acid aqueous solution (i.e.: magnitude of the aqueous solution as shown in Figure $4 \mathrm{~b}$ is larger than that of Figure $6 a)$. However, as Figure $6 \mathrm{~b}$, shows the electrode is relatively stable, retaining $90 \%$ of its capacity after 300 cycles. As shown in Rohl et. al [18], hexafluorophosphate has a relatively large volume as compared to smaller salts such as sulfates, occupying about $53 \AA^{3}$. The reduced performance in organic electrolyte as compared to aqueous sulfuric acid electrolyte may be due to the difference in size of molecules that insert themselves into voids left by oxygen vacancies. Similarly, larger salts can also contribute to faster capacity fade.

\section{CONCLUSIONS}

While current technology is limited to either a two-step deposition process or processes with poor conformality such as sputtering, we have achieved conformal and precise loading of TiN onto high-aspect ratio, porous CNT forests for supercapacitor applications. Analysis of our TiN-CNT architecture shows excellent performance due to increased oxygen vacancies in the native oxide layer, with the ability to further increase performance by taking advantage of the flexibility offered by the precise ALD process. Further work is needed on tradeoffs between chemical degradation due to irreversible oxidation and physical degradation due to larger molecule sizes of insertion ions in organic electrolytes. 


\section{ACKNOWLEDGEMENTS}

This material is based upon work supported by the National Science Foundation Graduate Research Fellowship under Grant No. 1106400. The authors would like to thank Ryan Rivers from the University of California, Berkeley Marvell Nanofabrication Lab and Michelle Rincon from the Stanford Nanofabrication Lab for their assistance, as well as all the members of the Liwei Lin Lab.

\section{REFERENCES}

[1] Y. Jiang, L. Lin, A Two-Stage, Self-Aligned Vertical Densification Process for As-Grown CNT Forests in Supercapacitor Applications, Sens. Actuators A 188 (2012) 261-267.

[2] Y. Jiang, A. Kozinda, L. Lin, Flexible Energy Storage Devices Based on Carbon Nanotube Forests with Built-in Metal Electrodes, Sens. Actuators A 195 (2013) 224-230.

[3] C. Chan, H. Peng, G. Liu, K. Mcllwrath, X.F. Zhang, R. Huggins, Y. Cui, High-performance lithium battery anodes using silicon nanowires, Nature Nano 3 (2008) 31-35.

[4] R. Warren, F. Sammoura, K.S. Teh, A. Kozinda, X. Zang, L. Lin, Electrochemically Synthesized and Vertically Aligned Carbon Nanotube-Polypyrrole Nanolayers for High Energy Storage Devices, Sens. Actuators A 231 (2015) 65-73.

[5] Y. Jiang, P. Wang, X. Zang, Y. Yang, A. Kozinda, L. Lin, Uniformly Embedded Metal Oxide Nanoparticles in Vertically Aligned Carbon Nanotube Forests as Pseudocapacitor Electrodes for Enhanced Energy Storage, Nanoletters 13 (2013) 3524-3530.

[6] R. Warren, F. Sammoura, F. Tounsi, M. Sanghadasa, L. Lin, Highly Active Ruthenium Oxide Coating via ALD and Electrochemical Activation in Supercapacitor Applications, J. Mater. Chem. A 3 (2015) 15568-15575.

[7] G. Fiorentino, S. Vollebregt, F. D. Tichelaar, R. Ishihara, P.M. Sarro. 3D solid-state supercapacitors obtained by ALD coating of high-density carbon nanotubes bundles, in Micro Electro Mechanical Systems (MEMS), 2014 IEEE 27th International Conference on, 2014, pp. 342-345.

[8] Y. Qiu, K. Yan, S. Yang, L. Jin, H. Deng, W. Li. "Synthesis of size-tunable anatase TiO2 nanospindles and their assembly into anatase@ titanium oxynitride/titanium nitridegraphene nanocomposites for rechargeable lithium Ion batteries with high cycling performance." ACS nano 4 (2010) 6515-6526.

[9] M.S. Balogun, M. Yu, C. Li, T. Zhai, Y. Liu, X. Lu, Y. Tong. Facile synthesis of titanium nitride nanowires on carbon fabric for flexible and high-rate lithium ion batteries. J. Mater. Chem. A 2 (2014) 10825-10829.

[10] Y. Jun, W.H. Hong, M. Antonietti, A. Thomas, Mesoporous, 2D Hexagonal Carbon Nitride and Titnium Nitride/Carbon Composites, Adv. Mater. 21 (2009) 4270-4274.

[11] X. Lu, G. Wang, T. Zhai, M. Yu, S. Xie, Y. Ling, C. Liang, Y. Tong, Y. Li, Stabilized TiN Nanowire Arrays for High-Performance and Flexible Supercapacitors, Nanoletters, 12 (2012) 5376-5381.

[12] A. Achour, J.B. Ducros, R.L. Porto, M. Boujtita, E. Gautron, L. LeBrizoual, M.A. Djouadi, T. Brousse, Heirarchical nanocomposite electrodes based on titanium nitride and carbon nanotubes for micro-supercapacitors, Nano Energy, 7 (2013) 104-113. 
[13] H. Li, S, Zhang, Q. Zhong. Effect of nitrogen doping on oxygen vacancies of titanium dioxide supported vanadium pentoxide for ammonia-SCR reaction at low temperature. J. of Coll. and Interface Sci. 402 (2013) 190-195.

[14] Y.Q. Jiang, Q. Zhou, L. Lin, Planar MEMS Supercapacitor Using Carbon Nanotube Forests", in Micro Electro Mechanical Systems (MEMS), 2009 IEEE 22nd International Conference (2009) 587-590.

[15]B. V. Tilak, C-P. Chen, S. K. Rangarajan, A model to characterize the impedance of electrochemical capacitors arising from reactions of the type $\mathrm{O}$ ad $+\mathrm{n} \mathrm{e}-\leftleftarrows \mathrm{R}$ ad. J. of Electroanal. Chem. 324 (1992) 405-414.

[16] G. Pacchioni, Numerical Simulations of Defective Structures: The Nature of Oxygen Vacancy in Non-reducible (MgO, SiO2, ZrO2) and Reducible (TiO2, NiO, WO3) Oxides, in: Defects at Oxide Surfaces, Springer International Publishing, 2015, pp. 1-28.

[17] A.K. Rumaiz, J. C. Woicik, E. Cockayne, H. Y. Lin, G. Hassnain Jaffari, S. I. Shah. Oxygen vacancies in $\mathrm{N}$ doped anatase TiO2: Experiment and first-principles calculations. Appl. Phys. Letters 95 (2009) 262111.

[18] A.L. Rohl, D. Michael, P. Mingos. The size and shape of molecular ions and their relevance to the packing of the hexafluorophosphate salts. J. Chem. Soc., Dalton Trans. 24 (1992) 3541-3552. 


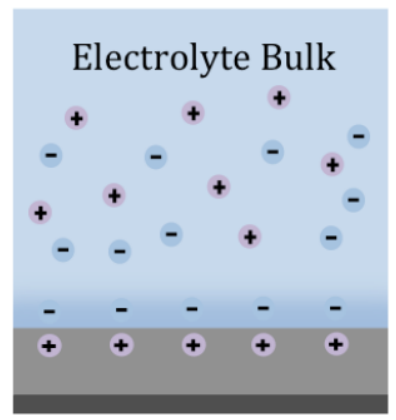

Electric Double Layer Capacitance

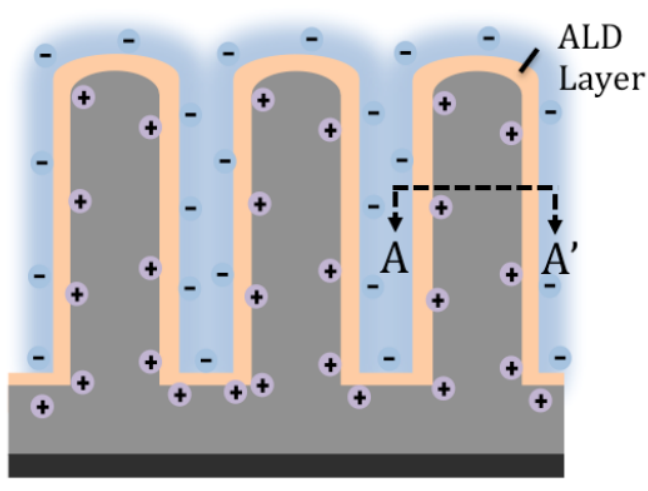

$$
\begin{gathered}
\text { Increasing surface area } \rightarrow \\
\text { Increasing capacitance }
\end{gathered}
$$

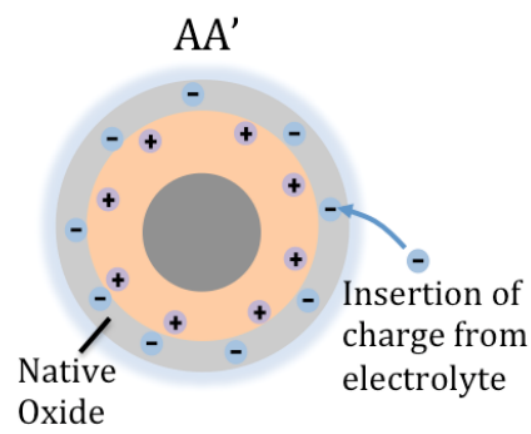

psuedocapacitance; oxygen vacancies arising from $\mathrm{TiO}_{2-x} \mathrm{~N}_{x}$ native oxide layer

Figure 1 Conceptual illustration of increasing capacitance through increased surface area and the pseudo capacitive effect. The storage and release of electrical charge occurs by adsorption and desorption of charge on the surface of TiN ("Double Layer charge storage") as well as oxidation of the surface layer. This surface layer of $\mathrm{TiO}_{2-\mathrm{x}} \mathrm{N}_{x}$, which forms as a native oxide layer, has a high nitrogen concentration, with a large number of oxygen vacancies in the surface. Both charge storage mechanisms combine to increase capacitance in the device. The current then flows through the ALD TiN and CNTs.

a)

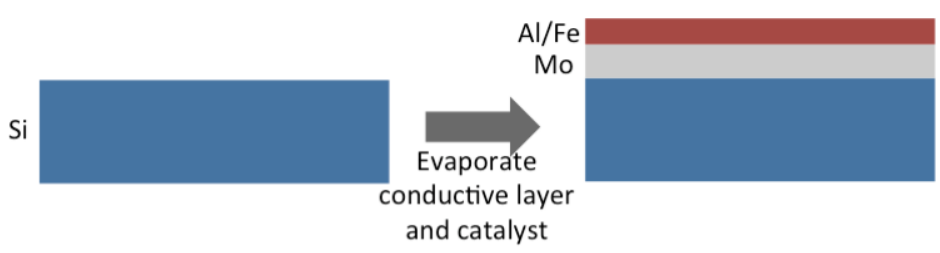

d)

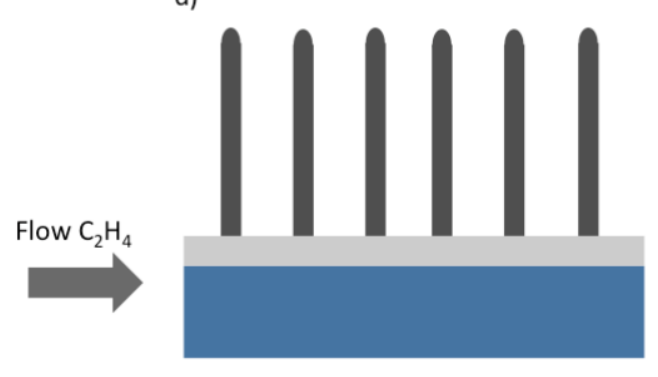

e)

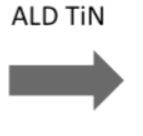

c)

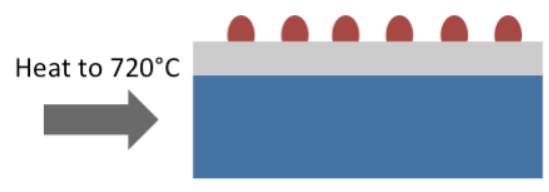

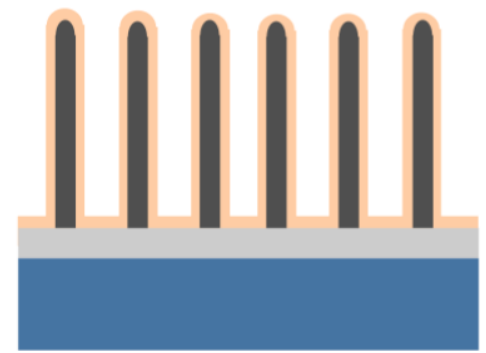

Figure 2 Process flow of substrate fabrication. Steps a-c show preparation of the CNT substrate before growth. This is carried out by evaporating a conductive layer and catalyst onto a silicon substrate with thermally grown oxide. When heated, the catalysts form droplets, which form the basis for CNT growth. In an ethylene environment, a vertically aligned CNT forest is grown by CVD (step d). Finally, in step e, TiN is deposited conformally onto the CNT forest using ALD. 


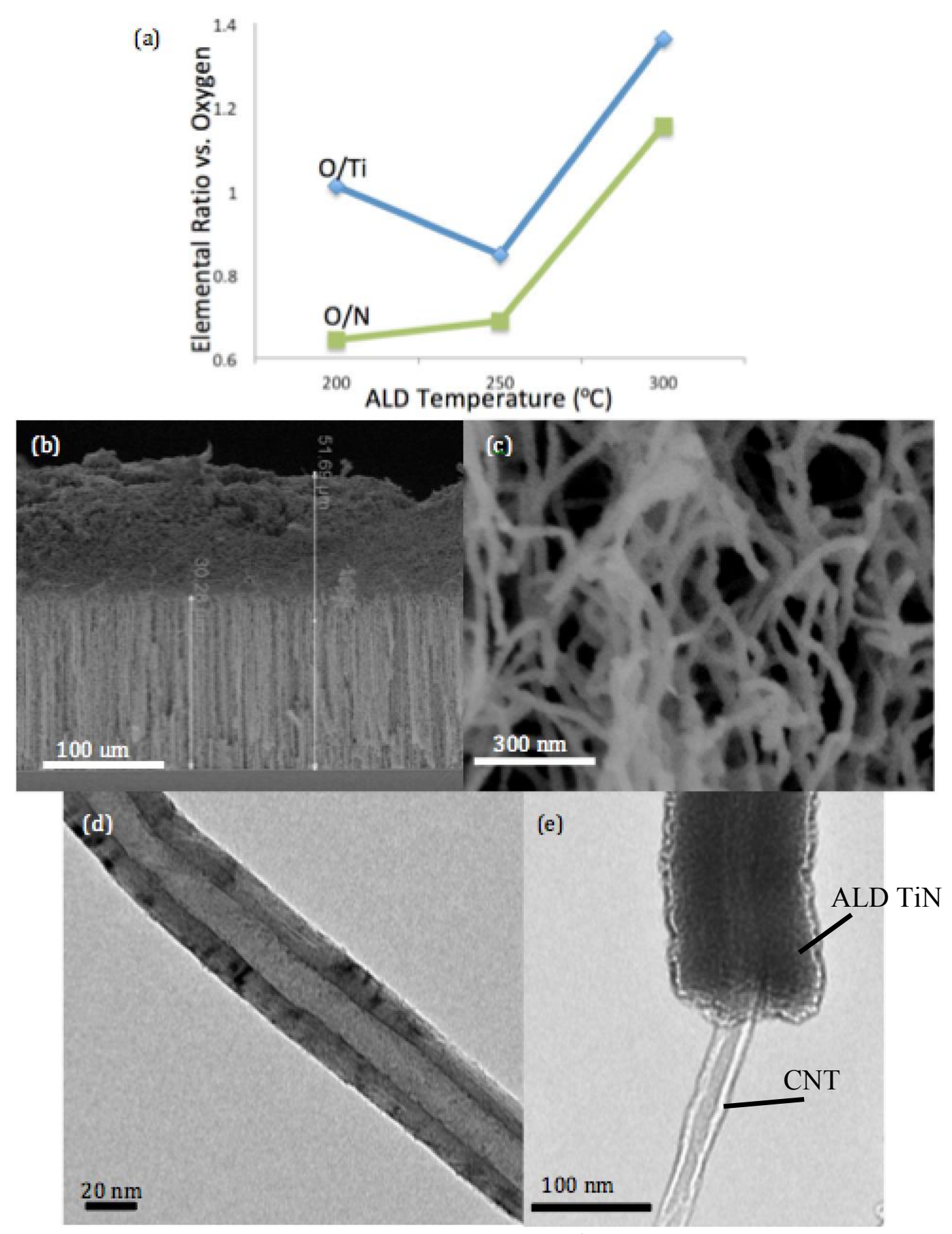

Figure 3 (a) XPS characterization of ALD TiN. Process temperature $250^{\circ} \mathrm{C}$ was chosen. (b) SEM image of uncycled ALD TiN coated at $250^{\circ} \mathrm{C}$ onto vertically aligned CNT forest. The forest is roughly 30 microns in height. (c) Close-up SEM image of uncycled ALD TiN coated CNTs showing conformal coverage of the ALD TiN. (d) TEM image of single multiwalled carbon nanotube as grown according to process outlined in Figure 2. (e) TEM image of ALD TiN coated multiwalled carbon nanotube showing conformal coverage and layer of about $20 \mathrm{~nm}$. 
(a)

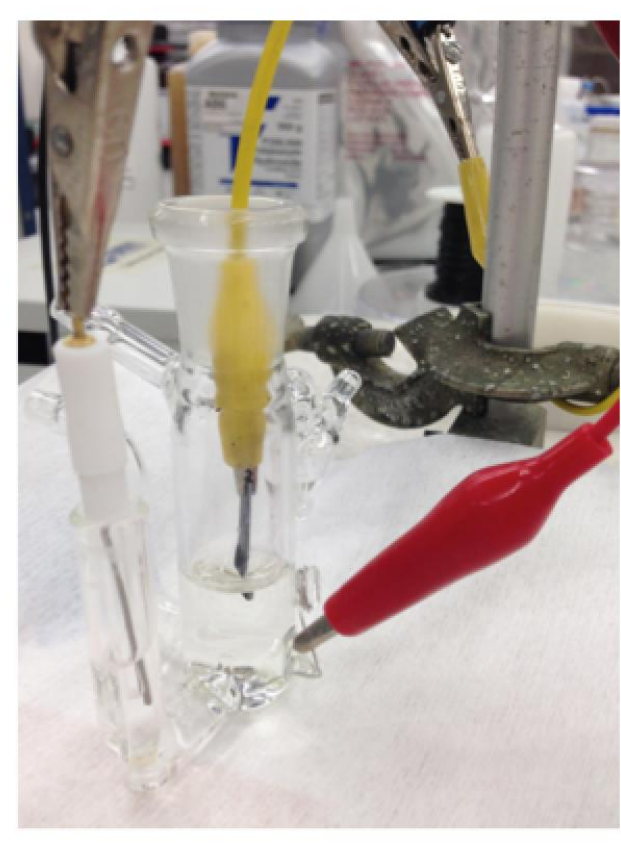

(b)

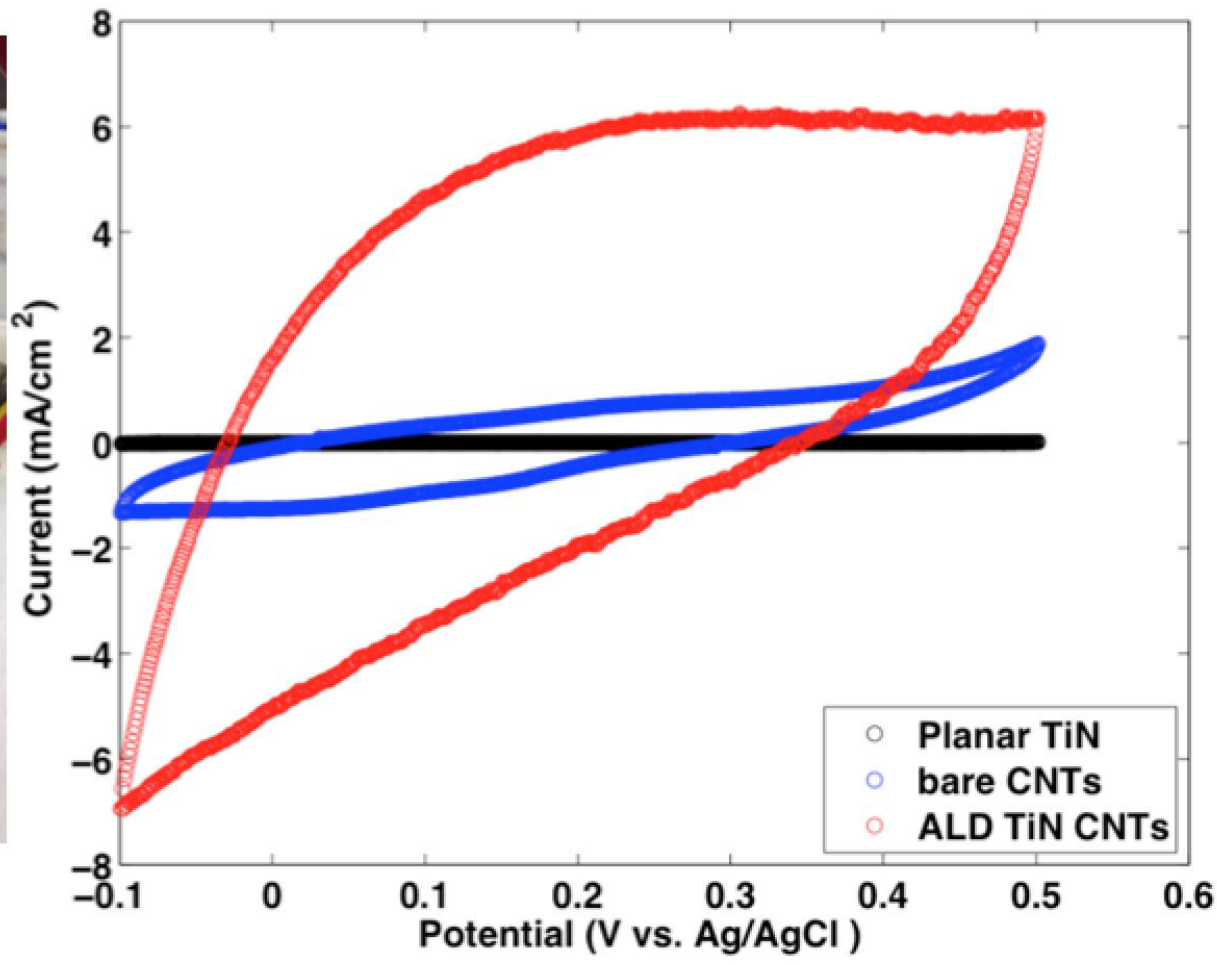

Figure 4 (a) Three electrode setup, with Pt wire as counter electrode vs. Ag/AgCl. (b) Cyclic Voltammograms (CVs) of ALD TiNCNTs as compared with bare CNTs and planar TiN, corresponding to $81 \mathrm{mF} / \mathrm{cm}^{2}, 14 \mathrm{mF} / \mathrm{cm}^{2}$, and $0.2 \mathrm{mF} / \mathrm{cm}^{2}$, respectively. CVS tested vs. $\mathrm{Ag} / \mathrm{AgCl}$. 
(a)

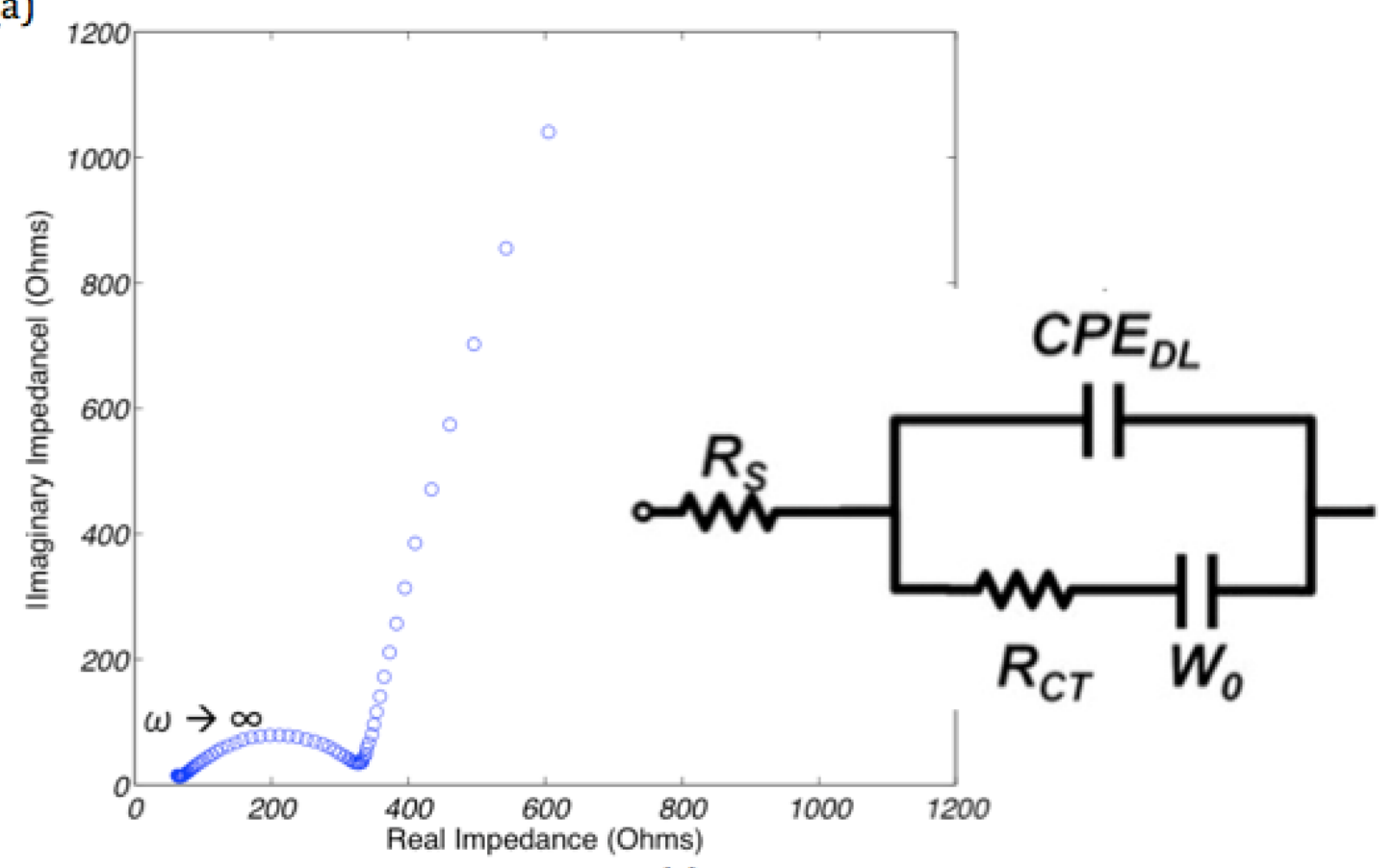

(b)

(c)
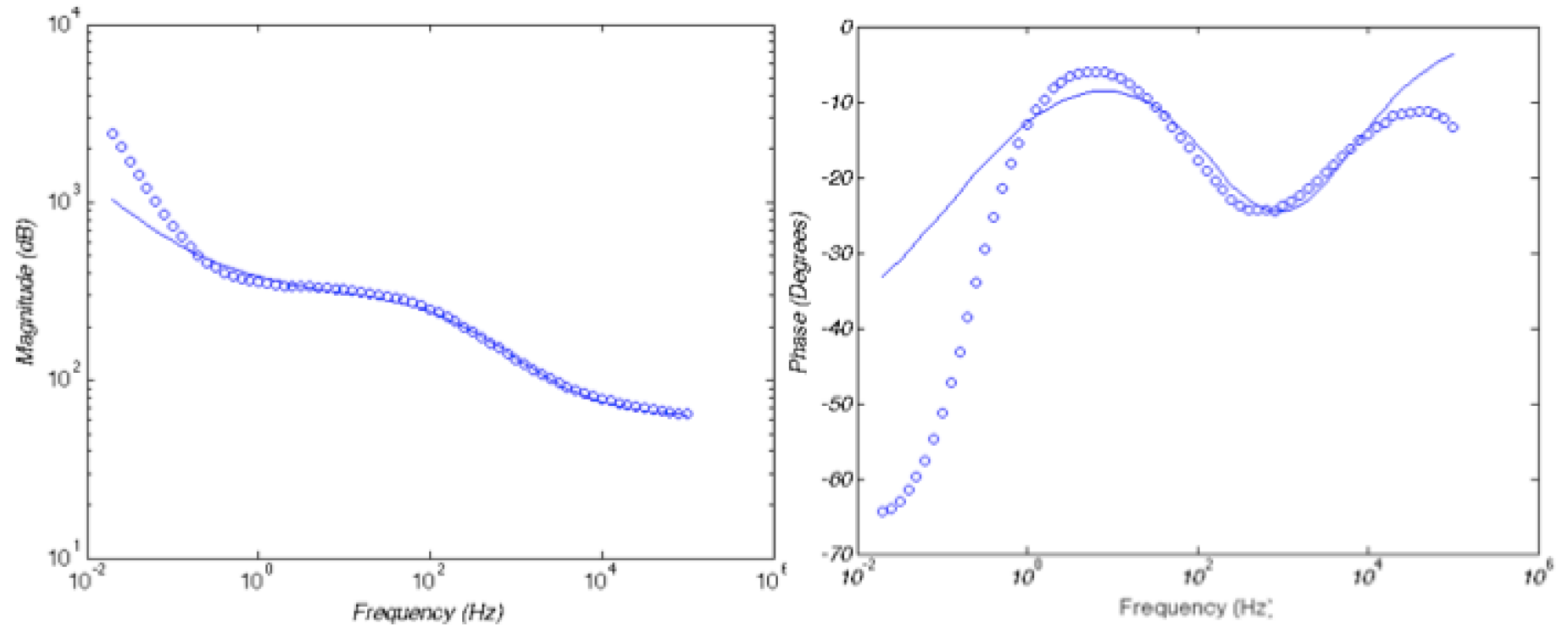

Figure 5 (a) Nyquist plot of Electrochemical Impedance Spectroscopy (EIS) for ALD TiN-CNTs in aqueous solution. The two regimes, corresponding to double layer capacitance effect and the diffusion limited region, show both pseudocapacitance as well as electric double layer capacitance. (inset) first order model of Randle's cell to model pseudocapacitive devices. (b)(c) Bode plot of ALD TiN-CNTs with Randle's cell fit. At lower frequencies, the fit estimates poorly (underestimates in Magnitude, overestimates in Phase). This is due to the use of an ideal Warburg impedance in the model. 
a)

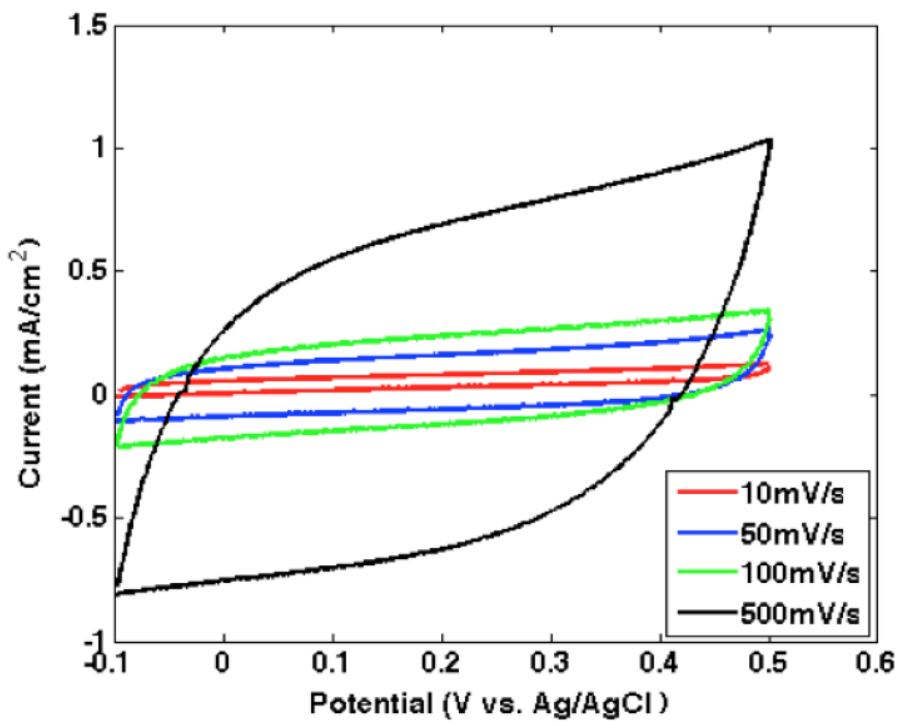

b)

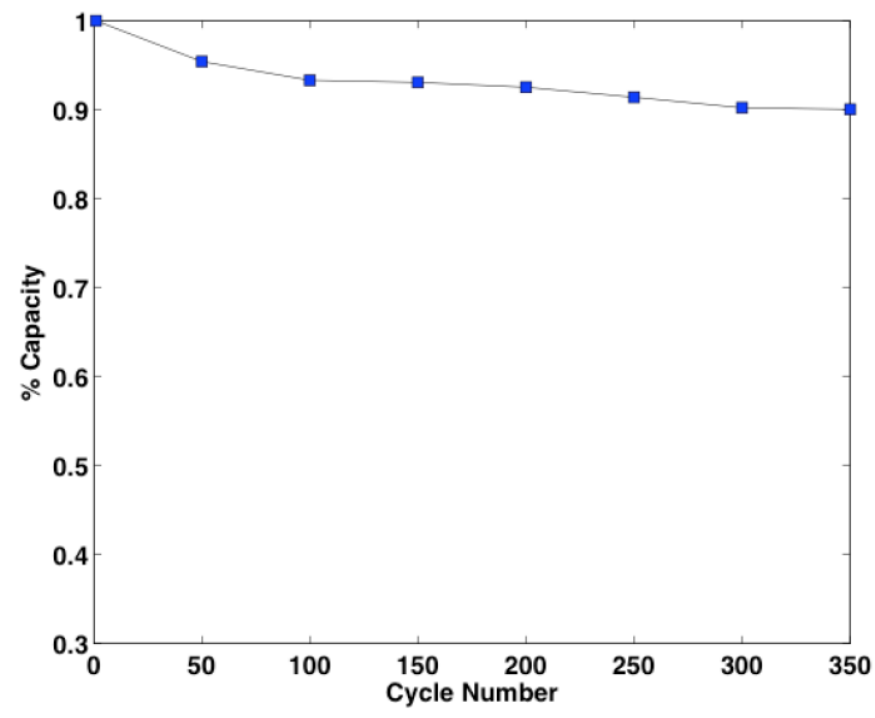

Figure 6 (a) Cyclic Voltammograms (CVS) of ALD TiN-CNTS in an organic electrolyte, 0.1M Tetrabutylammonium hexafluorophosphate in acetonitrile at varying scan rates, vs. $\mathrm{Ag} / \mathrm{AgCl}$. (b) Capacity fade over 350 cycles of ALD TiN-CNT electrode in organic electrode. 


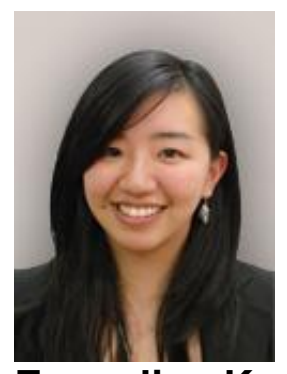

Emmeline Kao is a Ph.D. candidate and graduate student researcher working under Professor Liwei Lin in the Mechanical Engineering Department at University of California, Berkeley. She received her B.S.E. in Mechanical and Aerospace Engineering from Princeton University, and her M.S. in Mechanical Engineering from U.C. Berkeley. Her research focuses on energy storage and high surface area materials.

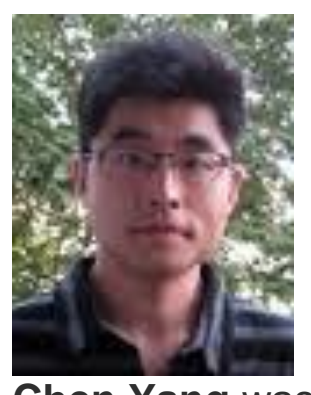

Chen Yang was born in Gansu, China, in 1981. He received his B Eng degree from Department of Electronic Engineering, Tsinghua University, Beijing, China, in 2003. Then he joined Division of Micro/Nano Devices and Systems, Institute of Microelectronics, Tsinghua University, where he is currently working toward the $\mathrm{PhD}$ degree on microelectronics and solid-state electronics. His research interests include new materials, integrated circuits and microelectronic devices for high-frequency applications.

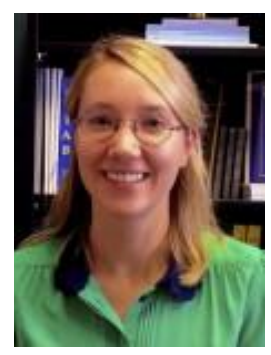

Roseanne Warren is a Ph.D. candidate and graduate student researcher working under Prof. Liwei Lin in the Mechanical Engineering Department at the University of California, Berkeley. Her research is focused on high surface area nanomaterials for energy generation and storage. Roseanne received her B.S. (2008) and M.S. (2009) degrees in Mechanical Engineering at Stanford University. In January 2016, Roseanne will be starting as an Assisstant Professor in the Mechanical Engineering Department at the University of Utah. 


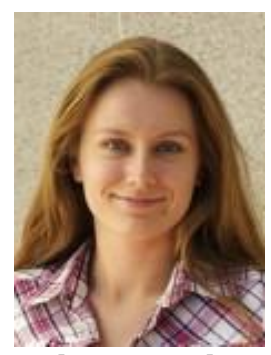

Alina Kozinda earned a Ph.D. in Mechanical Engineering from the University of California, Berkeley and a B.S. in both Mechanical Engineering and Mathematics at the University of Florida. When she isn't researching new ways to store energy in supercapacitors and lithium ion batteries using carbon nanotube films, she loves to be outdoors rock climbing and hiking, and indoors drawing and painting.

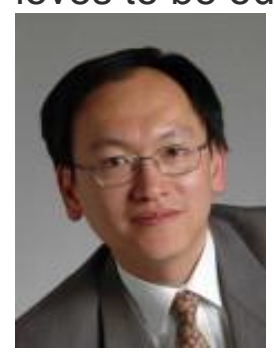

Liwei Lin received a B.S. degree in power mechanical engineering from National Tsing Hua University, Hsinchu, Taiwan, in 1986, and the M.S. and Ph.D. degrees in mechanical engineering from the University of California at Berkeley (UC Berkeley) in 1991 and 1993, respectively. He joined UC Berkeley in 1999 and is currently a professor in the Mechanical Engineering Department and co-director of the Berkeley Sensor and Actuator Center. His research interests include MEMS/NEMS devices for energy and biomedical applications. 